\title{
Design of vibration analysis and fault diagnosis for the rotating machinery based on virtual instrument
}

\author{
Haitao Bao ${ }^{1, \text { a }}$, Xing Zhao ${ }^{2, b}$, and Yu Yuan ${ }^{3, c}$ \\ ${ }^{1}$.School of Mechanical Engineering ,Dalian Jiaotong University,Dalian 116028,China \\ 2 .School of Mechanical Engineering ,Dalian Jiaotong University,Dalian 116028,China \\ ${ }^{3}$.School of EMU Application \& Maintenance Engineering ,Dalian Jiaotong University,Dalian \\ 116028, China \\ a1242434030@qq.com, bzhaoxing428@163.com, yuanyu_knife@163.com
}

Keywords: rotating machinery, envelopment analysis, cepstrum analysis ,orbit analysis , holo-spectrum analysis ,database

Abstract. Rolling bearings are widely used on the rotating machinery, but also the most vulnerable parts to damage. This paper designs a rolling bearing fault diagnosis system based on Labview. First of all, by using the transducer, such as torque transducer, temperature transducer, eddy current sensor and photoelectric sensor, to carry on the real-time controlling and display the signal in the special domain and in the time domain, in the meantime, the system can store data. Then the next part is capably of processing data by using the methods of filter analysis, envelopment analysis, cepstrum analysis, orbit analysis and holo-spectrum analysis. Finally the system is able to create database and provide an easy method for storing and manipulating the data which is measured by transducer and processed by the system.

\section{Introduction}

Now the rotating machinery plays an important role and get a high proportion of the mechanical equipment. And the rotating machinery have to use a common part called rolling bearing which can be damaged easily because of bad working condition or inadequacy lubrication. The reason why rotating machinery have fault is that the rolling bearing has damaged and it can't work well. If the bearing have fault, it will be the waster early and stimulates the resonance of common parts in the mechanical system later. Because of the special using environment of bearing, its life discreteness compared with other mechanical common parts is huge. Now we can't make accurate prediction of how long will it last. So it will be hard to change bearing by the way of estimating the life of rolling bearing. Because of the such character of it, we need real-time monitoring and fault diagnosis for the important application of rolling bearing to reduce generation of the hard mechanical fault. ${ }^{[1-2]}$

\section{System composition and Working principle}

The system consists of real-time collection system, off-live fault diagnosis system and fault information database. It mostly realizes function included rotating machinery condition monitoring signal detection and fault diagnosis. The system's program structure diagram is fig 1.

Every module of system briefly illustration:

Database collection of vibration signal equipment: Using acceleration sensor, eddy current sensor, photo electric sensor, temperature sensor and torque sensor measure the awaiting parts.

Real-time indication: Acquisition finished the database collection measured by every sensors. It will make them to host computer which can real-time display in the time and frequency domain and automated store the information. 


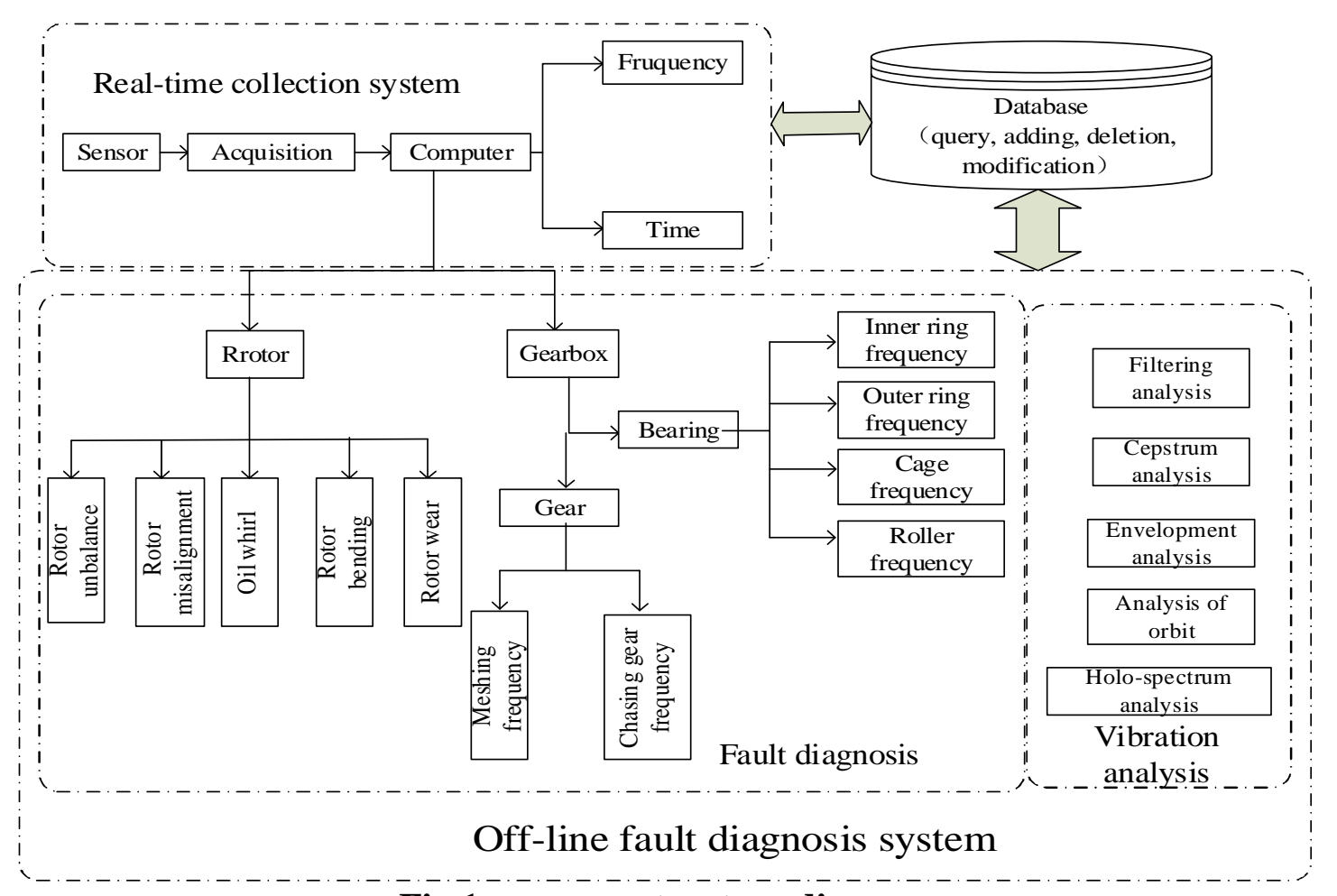

Fig 1 program structure diagram

Fault diagnosis: Through the calculation of the characteristic frequency for rotor, bearing and gear. We can compare with fault frequency and extract the character of fault to judge the type of it.

Database: The system has basic function of query, adding, deletion and modification and stores real-time collection database fault information and monitoring status.

Vibration analysis: Reading real time database, through real-time diagram and time-frequency spectrum, we can know the dynamic information of equipment. By the function of off-line fault diagnosis such as filtering analysis, cepstrum analysis, envelopment analysis, analysis of orbit and holo-spectrum analysis. We can analysis the real-time signal.

\section{Hardware part}

When we measure the awaiting parts by sensors. We totally use temperature sensor, eddy current sensor, torque sensor, photoelectric sensor, acceleration sensor and current transducer. They can make the parameters of mechanical vibration to output signal. Then we can transport the signal collected by sensor to computer to make off-line fault diagnosis.

The hardware part is the vibration analysis of rotating machinery and the experiment platform of fault diagnose QPZZ- II. The system QPZZ- II can simulate much vibration and state of rotating machinery. Also it will compare the analysis and the diagnosis of every state. The sampling system use the NI9234, NI9401, NI9219 and NI9237 produced by NI. They separately sample the signal of temperature sensor, eddy current sensor, torque sensor, photoelectric sensor and acceleration sensor. Fig 2 is the picture of the hardware part.
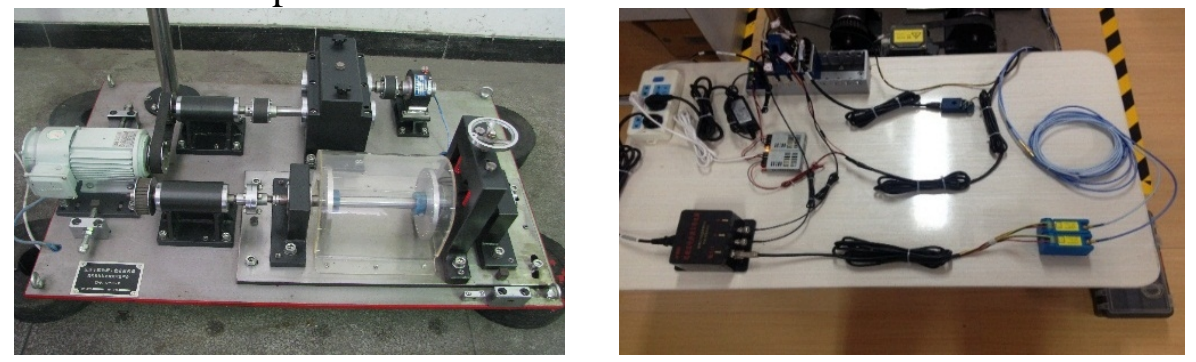

Fig 2 hardware part 


\section{Software part}

The mechanic components generate stationary signal when the operation is normal. The signal frequency mainly consists of two parts: the fundamental frequency of these components and noise frequency produced by external vibration source. Once faults occur, the amplitude and frequency components of the signal will change, through a variety of calculation 、 analysis processing and feature extraction of vibration signal to diagnose the faults of rolling bearing .Common methods for signal analysis including time domain analysis 、 frequency domain analysis and time-frequency domain analysis and so on.

\section{Parameter setting and database building}

After measuring the signals ,it is delivering to analysis system to processing ,the system includes off-line fault diagnosis and database .As is shown in Fig 3, in the database, if there is a parameter which have been storied already, the custom can delete or modify it, otherwise the custom need to type into some parameters like rotating speed, and the system will obtain the characteristic frequency, finally, all above information will be recorded to database. Database building is very useful to manage information and monitor bearing's condition. The results are objective and accurate.

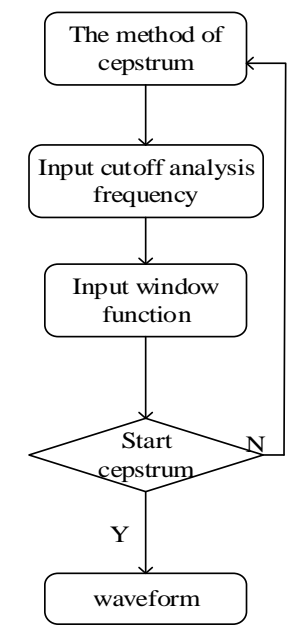

a Cepstrum analysis

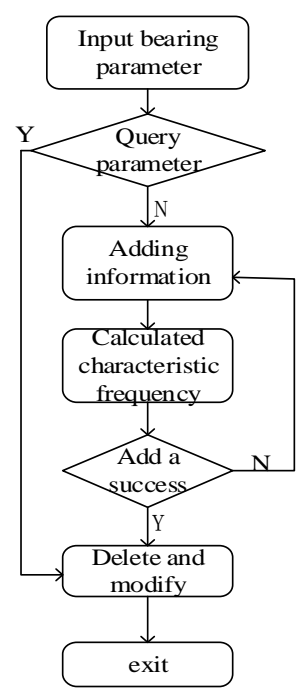

b Database

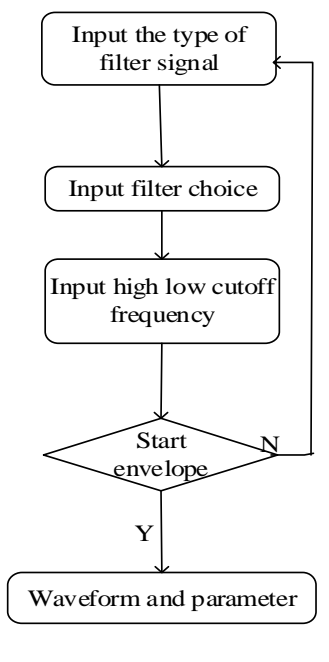

c Envelopment analysis

Fig 3 The idiographic flow of software part

Before analyzing the vibration signals, there are several parameters need to be set, the specific conditions of the signal can be intercepted according to the setting parameters, by that way, the system can avoid the interference caused by the early equipment operation instability and other external factors.

\section{The analysis of resonance-demodulation}

Resonance-demodulation is one of available methods for rolling bearing failure signal diagnosis .By using demodulation technique, the information of signal can be obtained effectively even under noise. The fault location can be pointed out by frequency spectrum analysis of these demodulated signals. The principle of resonance demodulation diagnosis is that, the rolling bearing has to interact with other components during operation process, if the surface of bearing components have damaged, the system will produce pulse signal. Due to the wide frequency band of the pulse signal, the high frequency of vibration system will be provoked.

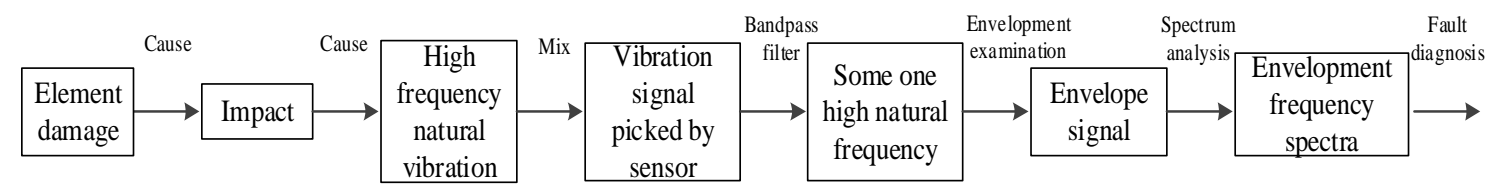

Fig 4 The analysis principle of resonance-demodulation 
Resonance demodulation method includes three steps (the fig 4 is the analysis principle of resonance-demodulation). At the first step, through bandpass filter (whose center frequency is equal to the natural frequency ), obtaining the natural frequency information ; Then by envelope demodulation method, the vibration signal can be separated from intrinsic frequency of the system (the process of envelop demodulation have been shown in Fig 6); The last step, using the low frequency filter to remove the high frequency noise and carrying on spectrum analysis .The method is high efficient and convenient to diagnosis the faults of bearing. The specific program has been shown in Fig 5.

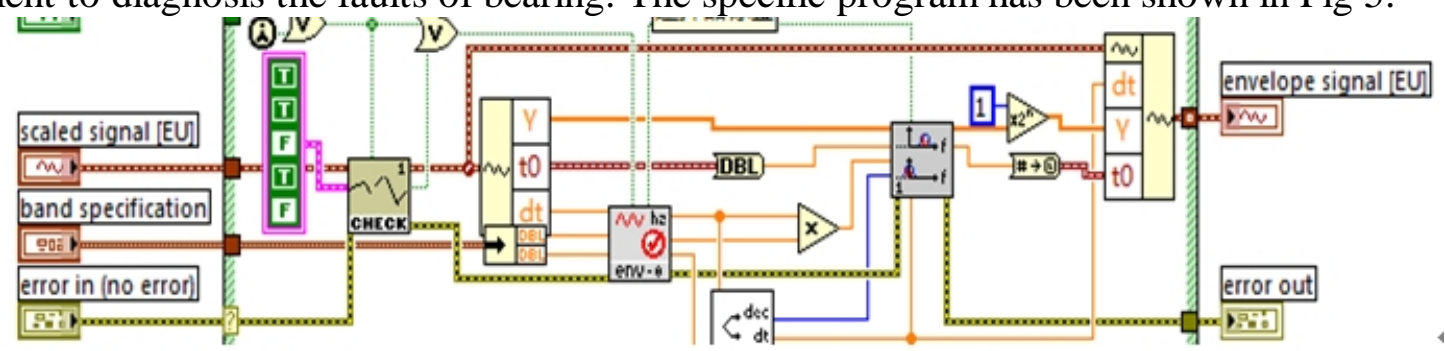

Fig 5 The specific program of resonance-demodulation

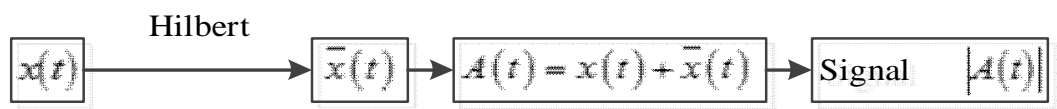

Fig 6 The process of envelop demodulation

Besides, this system consists of filtering analysis, cepstrum analysis and holo-spectrum analysis .Due to space limitation, studies on above parts cannot be presented in detail. As is shown in Fig 7 is part of the front panel of the system
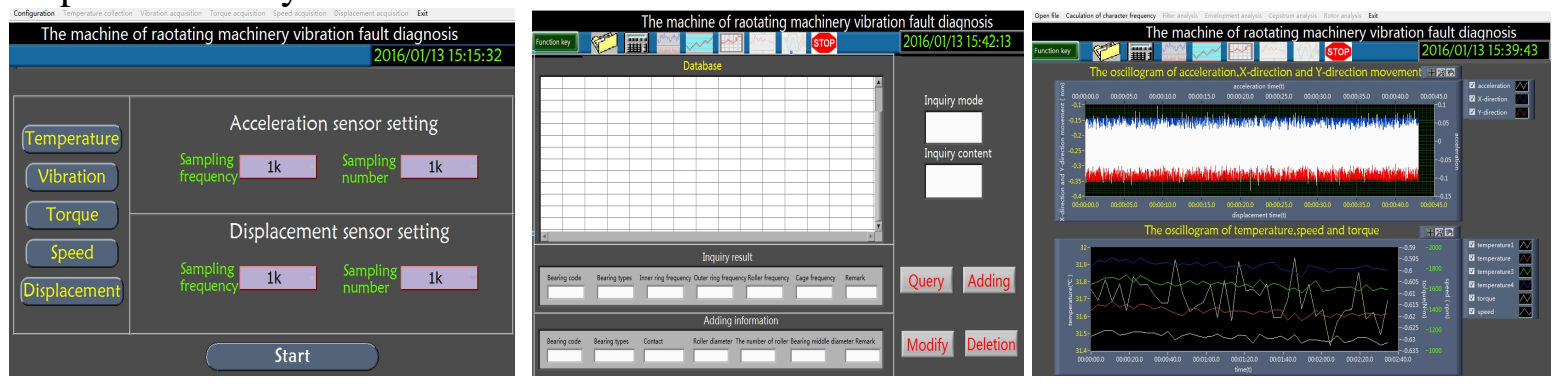

Fig 7 The front panel of the system

\section{Conclusions}

The major function of system: Through varies of sensors and acquisition, it takes the real-time database of the rotating machinery await measuring common parts to host computer. After that, it will get database into fault analysis and calculation of character frequency. Database can query, adding and deletion for basic parameter of bearing. The result of fault diagnosis and condition monitoring.

\section{References}

[1] Silva,J. L. H. ,Marqucs Cardoso,A. J. Bearing failures diagnosis in thrcc-phase induction motors by extended park's vector approach[C]. Industrial Electronics Society, 2005. IECON 2005. 32nd Annual Conference of IEEE 6-10 Nov. 2005 Page(s): 2585-2590

[2] Fu,Z. Brown,D. J. Haynes,B. P. A new method of non-stationary signal analysis for control motor bearing fault diagnosis[J]. Intelligent Signal Processing. 2003 IEEE International Symposium on 4-6 Sept. 2003 Page(s): 99-104 